\title{
Competencias digitales docentes y estrategias de aprendizaje en la formación del profesional de educación física
}

Teaching digital skills and learning strategies in the training of physical education professionals

1 Edison Andrés Castro Pantoja iD https://orcid.org/0000-0002-4606- 5016 Universidad Técnica de Ambato, Carrera de Pedagogía de la Actividad Física y Deporte. Tungurahua, Ecuador ea.castro@uta.edu.ec

2 Anthony Paúl Sandoval Gordón (iD) https://orcid.org/0000-0002-4461-1896 Universidad Técnica de Ambato, Carrera de Pedagogía de la Actividad Física y Deporte. Tungurahua, Ecuador pau1100@hotmail.es

3 Lenin Esteban Loaiza Dávila https://orcid.org/0000-0002-5769-2795. Universidad Técnica de Ambato, Carrera de Pedagogía de la Actividad Física y Deporte. Tungurahua, Ecuador e.loaiza@uta.edu.ec

4 Christian Mauricio Sánchez Cañizares (iD) https://orcid.org/0000-0002-0677-7246 Universidad Técnica de Ambato, Carrera de Pedagogía de la Actividad Física y Deporte. Tungurahua, Ecuador cm.sanchazc@uta.edu.ec

Artículo de Investigación Científica y Tecnológica Enviado: 24/12/2021 Revisado: 29/12/2021 Aceptado: $12 / 01 / 2022$ Publicado:08/03/2023

DOI: https://doi.org/10.33262/concienciadigital.v6i1.4.2048

Cítese: Castro Pantoja, E. A., Sandoval Gordón, A. P., Loaiza Dávila, L. E., \& Sánchez Cañizares, C. M. (2023). Competencias digitales docentes y estrategias de aprendizaje en la formación del profesional de educación física. ConcienciaDigital, 6(1.4), 1031-1045. https://doi.org/10.33262/concienciadigital.v6i1.4.2048

CONCIENCIA DIGITAL, es una Revista Multidisciplinar, Trimestral, que se publicará en soporte electrónico tiene como misión contribuir a la formación de profesionales competentes con visión humanística y crítica que sean capaces de exponer sus resultados investigativos y científicos en la misma medida que se promueva mediante su intervención cambios positivos en la sociedad. https://concienciadigital.org

La revista es editada por la Editorial Ciencia Digital (Editorial de prestigio registrada en la Cámara Ecuatoriana de Libro con No de Afiliación 663) www.celibro.org.ec 
Palabras claves: competencias digitales docentes, estrategias de aprendizaje.

Keywords:

teaching digital competencies, learning strategies.
Resumen

Introducción. El nivel de competencias digitales docentes delimita el nivel de aplicación de estrategias de aprendizaje en los estudiantes de formación en el área de Educación Física, ya que el conocimiento y manejo de la tecnología e informática hace que el docente, presente maneras dinámicas y diversas de enseñar. Objetivo. Se planteó determinar el grado de incidencia del nivel de competencias de carácter digital en un grupo de docentes sobre la aplicación de estrategias de aprendizaje enunciadas por los estudiantes universitarios de formación en Educación Física. Metodología. Estudio de enfoque cuantitativo de tipo de investigación no experimental, de alcance correlacional con un corte transversal, realizado a través de la técnica de la encuesta específicamente 2 cuestionarios validados estadísticamente y por criterio de expertos (Cuestionario de competencias digitales docentes y cuestionario de estrategias de aprendizaje) aplicados en una muestra de 25 docentes y cada uno con un grupo de estudiantes universitarios en formación de Educación física, con un total de 1002 individuos. Resultados. El mayor porcentaje de los docentes en estudio se encontraron en un nivel B2 de competencias digitales y sus estudiantes determinaron que estos se encontraban en un nivel alto de aplicación de estrategias de enseñanza, obteniendo una relación estadística entre las variables en un nivel de $\mathrm{P}<0.05$. Conclusión. El análisis y recolección de datos concluyeron que, efectivamente aquellos docentes con un nivel competencial digital avanzado, poseen un nivel alto de aplicación de estrategias de aprendizaje en los estudiantes universitarios de formación en educación física.

\section{Abstract}

Introduction. The level of teachers' digital competences delimits the level of application of learning strategies in the students of Physical Education since the knowledge and management of technology and informatics makes the teacher present dynamic and diverse ways of teaching. Objective. It was proposed to determine the degree of incidence of the level of digital competences in a group of teachers on the application of learning strategies stated by university students of Physical Education. Methodology. Study of quantitative approach of non-experimental research type, of correlational scope with a cross-sectional cut, carried out through 
the survey technique specifically 2 questionnaires statistically validated and by expert criteria (Questionnaire of teachers' digital competences and questionnaire of learning strategies) applied in a sample of 25 teachers and each one with a group of university students in training of Physical Education, with a total of 1002 individuals. Results. The highest percentage of the teachers under study were found to be at a B2 level of digital competencies and their students determined that these were at a high level of application of teaching strategies, obtaining a statistical relationship between the variables at a level of $\mathrm{P}<0.05$. Conclusion. The analysis and data collection concluded that, indeed those teachers with an advanced digital competence level, have a high level of application of learning strategies in university students of physical education training.

\section{Introducción}

La educación superior ha surgido grandes cambios a través del tiempo, pero sin duda la pandemia ha marcado un antes y un después en este proceso, obligando a cambiar totalmente la forma de enseñanza, surgiendo nuevos requerimientos formativos en los diferentes niveles de formación y carreras universitarias. La sociedad actual está marcada por el desarrollo constante de la ciencia y la tecnología, que cada minuto descubre nuevas tecnologías y un progresivo avance científico - tecnológico, promoviendo la innovación didáctica de los docentes. El docente está obligado a plantear ideas creativas e innovadoras para optimizar el proceso enseñanza aprendizaje, a través de estrategias basadas en el nivel de competencias digitales que estos poseen (Addine et al., 2020). El desarrollo social y profesional se fundamente por la adquisición de diferentes competencias entre ellas las de carácter digital que permiten crear y adaptar el proceso educativo en nuevos escenarios de aprendizaje, potenciando una transformación educativa de calidad, que contribuya a la preparación de futuros profesionales calificados y competentes (Del Rocío et al., 2018).

Las competencias digitales en el ámbito profesional se desarrollan entorno a el saber hacer, saber estar y saber ser (Sandoval \& Guajardo, 2017). Las competencias son utilizadas por los profesionales en dependencia de diferentes situaciones específicas, las tareas a resolver y los escenarios de desenvolvimiento (Ramírez-García, 2018).

Las demandas de la época determinan el manejo de las diferentes competencias digitales, que permiten desarrollas y aplicar estrategias de enseñanza basadas en las necesidades y capacidades de los estudiantes consiguiente (Martínez et al., 2020). En 
la actualidad la educación superior se basa en una formación holística por competencias, encaminadas a desarrollar el interés, la responsabilidad y el deseo de preparación de los estudiantes (Casanova, 2018). La constante formación del docente promueve el aprendizaje autónomo del estudiantado, incentivando a los procesos de investigación, encaminados a la resolución de problemas, por medio de acciones prácticas y el uso de herramientas tecnológicas, formando individuos competentes y profesionales (Suárez, 2020). La educación de calidad se consigue a través del grado de compromiso y el nivel de capacitación y desarrollo profesional (Loaiza-Aguirre \& Andrade-Abarca, 2021).

Según Hernández y Ortiz, (2019), a pesar de las tendencias de formación y capacitación, la preparación del docente tiende a ser reducida, pasiva, subordinada e instrumental. Por ello, sugiere iniciativas hacia una formación integral en la que se promuevan actividades que incentiven el papel activo del educando, así como la autonomía y la autorregulación del aprendizaje. En conclusión, la formación docente es continua y su oficio es interminable, luego su capacitación no termina al conseguir un diploma o al culminar un curso, sino más bien es ahí donde empieza el proceso de reflexión, autoevaluación y experimentación con el propósito de optimizar el aprendizaje (Dussel, 2020). En la actualidad los procesos de formación van direccionados al desarrollo de competencias de carácter digital basados en la necesidad de reformar el perfil profesional según los contextos del entorno educativo, desarrollando mejor las estrategias de aprendizaje específicos de cada formación (Otero et al., 2021).

Al hablar de competencias de carácter digital, estas involucran a la utilización de componentes tecnológicos, de comunicación, alfabetización digital, empleo de herramientas tecnológicas y su aplicación en distintas situaciones y asignaturas (Engen, 2019), enfocándose en un diseño e innovación de los contenidos digitales, que permitan la profesionalización docente (Cuartero et al., 2019), que pueda resolver dificultades en la práctica profesional respecto a la integración digital e incluso más aún en la innovación digital en diferentes contextos (Fernández y Pérez, 2018). La pandemia del Covid-19 permitió evidenciar la falta de desarrollo de competencias digitales, direccionados a la enseñanza virtual (Santos-Loor et al., 2021).

El marco común de competencia digital docente, elaborado por el Instituto Nacional de Tecnologías Educativas y Formación del Profesorado (INTEF, 2017), centra su atención en el diagnóstico y la mejora competencial digital del profesorado en 5 diferentes áreas competenciales: Información y alfabetización informacional, comunicación y colaboración, creación de contenidos digitales, seguridad y resolución de problemas, además de 21 competencias estructuradas, contenidos que permiten categorizar en 6 niveles desde el nivel A1 nivel - básico hasta el C2 nivel avanzado 
de conocimiento y aplicación (Cabero-Almenara \& Palacios-Rodríguez et al., 2020).

Una de las tendencias digitales aplicadas por el docente es el flipped classroom o aprendizaje invertido, ejecutado en base a la inversión de los papeles de la enseñanza a través de herramientas tecnológicas, modelo aplicable a la educación física. Otra tendencia digital, son los procesos de gamificación, considerada una metodología emergente, que permite la creación de juegos en contextos no lúdicos aplicables al proceso de enseñanza aprendizaje (Parra-González \& Segura-Robles, 2019). En base a las estrategias de aprendizaje es preciso mencionar que esta se refiere a un plan estructurado y ordenado cuidadosamente, mediante el cual se emplean todas las capacidades y medios para conseguir los objetivos propuestos, siendo un proceso caracterizado por promover la autonomía y la motivación del estudiante en sus acciones de formación (Al-Quraishi, 2021).

Sin importar el escenario educativo en el que se efectúe el proceso de enseñanzaaprendizaje, las estrategias aplicadas deben alejarse de lo tradicional, enfocándose en solucionar los procesos psicológicos de los estudiantes (Polo et al., 2020). En la educación superior, la didáctica moderna empeladas por los docentes debe ofrecer respuestas creativas y dinámicas ante los nuevos desafíos emergentes, puesto que, la incidencia de la tecnología en la educación, los modelos de enseñanza basados en el desarrollo de competencias y el intercambio informático digital, requieren el cambio de prácticas educativas, para promover el desarrollo de una cultura innovadora, creativa, pedagógica y didáctica que permita consolidar la calidad universitaria en todas las formaciones existentes (Macanchí, 2020).

Por tal razón se plantea como objetivo de la investigación determinar la incidencia del nivel de competencias docentes de carácter digital en la aplicación de estrategias de aprendizaje en estudiantes universitarios de formación en educación física.

\section{Metodología}

El estudio planteado responde a un enfoque de tipo cuantitativo, de tipo no experimental por alcance correccional y de corte transversal, desarrollado a través de los métodos analítico e hipotético deductivo.

Partiendo de una población finita no desconocida a través de un muestreo no probabilístico por voluntariado, se determinó de 25 docentes que de manera voluntaria accedieron a participar en el estudio, cada uno con su grupo de estudiantes que llegaron a un total de 1002.

Se aplicó la técnica de la encuesta y como instrumento de investigación el "Cuestionario de Competencias Digitales Docentes (CDD) conformado por 54 ítems, que evaluaron cinco dimensiones determinadas por el marco común de competencias 
digitales del INTEF (2017): información y alfabetización informacional, comunicación y colaboración, creación de contenido digital, seguridad y resolución de problemas, el instrumento fue verificado en su fiabilidad estadística con un coeficiente de 0.98 (Alpha de Cronbach) y validado por juicio de expertos.

El nivel de aplicación de estrategias empleadas para el aprendizaje se evaluó a través de un cuestionario construido en base al sustento teórico respecto a las herramientas empleadas por el profesorado para el aprendizaje según su competencia digital. El instrumento se basó en una escala de Likert de 5 niveles que reflejan el grado de acuerdo y desacuerdo, además paso por un proceso de validación estadística ( 0.95 de coeficiente de Alfa de Crombach) determinando su fiabilidad y un proceso de juicio de expertos para determinar su pertinencia.

Paquete estadístico SPSS versión 25: análisis de frecuencias y porcentajes para las variables cualitativas y descriptivo para las variables cuantitativas, pruebas de normalidad de Shapiro-Wilk (muestras docentes) y Kolmogorov-Smirnov (muestras estudiantes), la prueba no paramétrica U de Mann de Whitney para muestras independientes, así como la prueba Chi cuadrado de Pearson para la comprobación de la relación estadística entre las variables de estudio.

\section{Resultados}

Como todo proceso de investigación en primer lugar se realizó la caracterización de la muestra de estudio, al ser una investigación con dos muestras determinadas, en un inicio se planteó caracterizar a los docentes (tabla 1).

Tabla 1

Caracterización de la muestra de estudio docente

\begin{tabular}{|c|c|c|c|c|c|c|}
\hline \multirow{2}{*}{ Sexo } & \multirow{2}{*}{$\mathrm{f}$} & \multirow{2}{*}{$\%$} & \multicolumn{2}{|c|}{ Edad } & \multicolumn{2}{|c|}{ Años de experiencia docente } \\
\hline & & & $\mathrm{M}$ & $\overline{D S}$ & $\mathrm{M}$ & DS \\
\hline Masculino & 18 & 72.0 & 43.00 & 7.717 & 17.06 & 6.56 \\
\hline Femenino & 7 & 28.0 & 49.00 & 10.80 & 18.14 & 9.14 \\
\hline \multicolumn{3}{|c|}{$\mathrm{P}$} & \multicolumn{2}{|c|}{$0.302 *$} & \multicolumn{2}{|c|}{$0.760^{*}$} \\
\hline Total & 25 & 100.0 & 45.04 & 8.82 & 17.36 & 7.19 \\
\hline
\end{tabular}

Nota. Distribución de frecuencias (f) y porcentajes (\%) de la variable del sexo; valores medios (M) y desviaciones estándares (DS) de las variables de la edad y años de experiencia docente, sin diferencias significativas en un nivel de $\mathrm{P} \geq 0.05\left(^{*}\right)$.

La muestra de estudio presento un porcentaje superior en relación con los grupos por sexo, siendo el masculino casi 3 veces mayor. En relación con la edad y años de experiencia los grupos no presentaron diferencias significativas en un nivel de $\mathrm{P} \geq 0.05$.

Consiguientemente se planteó caracterizar a los grupos de estudiantes participantes en el proceso de enseñanza aprendizaje con cada docente (tabla 2). 


\section{Tabla 2}

Caracterización de la muestra de grupos de estudiantes por docente

\begin{tabular}{|c|c|c|c|c|c|c|c|c|c|}
\hline \multirow{3}{*}{ Grupos de estudiantes } & \multicolumn{4}{|c|}{ Masculino } & \multicolumn{4}{|c|}{ Femenino } & \multirow{3}{*}{$\mathrm{P}$} \\
\hline & \multirow{2}{*}{$\mathrm{f}$} & \multirow{2}{*}{$\%$} & \multicolumn{2}{|c|}{ Edad } & \multirow{2}{*}{$\mathrm{f}$} & \multirow{2}{*}{$\%$} & \multicolumn{2}{|c|}{ Edad } & \\
\hline & & & $\mathrm{M}$ & DS & & & $\mathrm{M}$ & DS & \\
\hline Estudiantes docente 1 & 30 & 3.8 & 21.9 & 2.2 & 10 & 4.7 & 21.0 & 1.2 & 0.280 \\
\hline Estudiantes docente 2 & 27 & 3.4 & 22.2 & 2.4 & 13 & 6.1 & 21.3 & 1.9 & 0.357 \\
\hline Estudiantes docente 3 & 33 & 4.2 & 22.8 & 2.4 & 8 & 3.8 & 22.1 & 1.9 & 0.451 \\
\hline Estudiantes docente 4 & 32 & 4.1 & 20.9 & 1.7 & 7 & 3.3 & 21.6 & 2.6 & 0.587 \\
\hline Estudiantes docente 5 & 34 & 4.3 & 24.0 & 4.1 & 7 & 3.3 & 22.4 & 2.1 & 0.507 \\
\hline Estudiantes docente 6 & 30 & 3.8 & 23.0 & 2.8 & 10 & 4.7 & 22.9 & 1.0 & 0.862 \\
\hline Estudiantes docente 7 & 34 & 4.3 & 22.9 & 4.3 & 5 & 2.3 & 23.6 & 1.9 & 0.298 \\
\hline Estudiantes docente 8 & 31 & 3.9 & 22.5 & 2.3 & 9 & 4.2 & 21.1 & 1.5 & 0.111 \\
\hline Estudiantes docente 9 & 29 & 3.7 & 22.8 & 2.8 & 11 & 5.2 & 21.2 & 1.3 & 0.032 \\
\hline Estudiantes docente 10 & 28 & 3.5 & 21.6 & 2.4 & 12 & 5.6 & 21.0 & 2.0 & 0.471 \\
\hline Estudiantes docente 11 & 34 & 4.3 & 22.6 & 2.3 & 6 & 2.8 & 22.3 & 2.1 & 0.758 \\
\hline Estudiantes docente 12 & 30 & 3.8 & 21.8 & 2.4 & 10 & 4.7 & 21.8 & 2.2 & 0.836 \\
\hline Estudiantes docente 13 & 33 & 4.2 & 22.2 & 3.5 & 7 & 3.3 & 22.3 & 2.3 & 0.471 \\
\hline Estudiantes docente 14 & 32 & 4.1 & 24.3 & 3.3 & 8 & 3.8 & 22.5 & 1.1 & 0.113 \\
\hline Estudiantes docente 15 & 34 & 4.3 & 21.8 & 3.6 & 6 & 2.8 & 23.0 & 1.5 & 0.156 \\
\hline Estudiantes docente 16 & 32 & 4.1 & 23.0 & 3.4 & 8 & 3.8 & 22.1 & 2.2 & 0.669 \\
\hline Estudiantes docente 17 & 32 & 4.1 & 23.0 & 2.8 & 9 & 4.2 & 21.4 & 1.5 & 0.064 \\
\hline Estudiantes docente 18 & 31 & 3.9 & 21.8 & 2.4 & 8 & 3.8 & 21.0 & 2.2 & 0.251 \\
\hline Estudiantes docente 19 & 27 & 3.4 & 22.2 & 2.4 & 13 & 6.1 & 20.9 & 1.3 & 0.154 \\
\hline Estudiantes docente 20 & 33 & 4.2 & 22.6 & 2.4 & 7 & 3.3 & 22.4 & 1.8 & 0.942 \\
\hline Estudiantes docente 21 & 30 & 3.8 & 21.0 & 2.9 & 10 & 4.7 & 21.6 & 2.4 & 0.199 \\
\hline Estudiantes docente 22 & 33 & 4.2 & 24.6 & 3.4 & 7 & 3.3 & 23.1 & 1.6 & 0.428 \\
\hline Estudiantes docente 23 & 34 & 4.3 & 21.7 & 2.8 & 6 & 2.8 & 23.0 & 1.1 & 0.152 \\
\hline Estudiantes docente 24 & 34 & 4.3 & 23.2 & 4.0 & 6 & 2.8 & 23.3 & 1.9 & 0.445 \\
\hline Estudiantes docente 25 & 32 & 4.1 & 23.1 & 2.9 & 10 & 4.7 & 21.0 & 1.6 & 0.023 \\
\hline Total & 789 & 100.0 & 22.6 & 3.1 & 213 & 100.0 & 21.9 & 1.9 & \\
\hline
\end{tabular}


Siguiendo la metodología planteada se valoró las diferentes dimensiones de competencias digitales en la muestra de docentes: Información y alfabetización informacional (IAI), comunicación y colaboración (CC), construcción de contenidos digitales (CCD), seguridad (SG), resolución de problemas (RP), índice de competencias digitales (CD) y nivel de competencias digitales (CD), resultados que se evidencian en la tabla 3.

Tabla 3

Valoración de las dimensiones de competencias digitales en la muestra de docentes

\begin{tabular}{cccccccc}
\hline CI & C-IAI & C-CC & C-CCD & C-SG & C-RP & I-CD & Nivel de CD \\
\hline 1 & 5 & 5 & 5 & 4 & 5 & 5 & C1 \\
2 & 5 & 5 & 4 & 4 & 3 & 4 & B2 \\
3 & 5 & 6 & 4 & 5 & 4 & 5 & C1 \\
4 & 3 & 3 & 3 & 3 & 2 & 3 & B1 \\
5 & 6 & 6 & 4 & 3 & 5 & 5 & C1 \\
6 & 4 & 4 & 3 & 3 & 4 & 4 & B2 \\
7 & 3 & 5 & 3 & 4 & 4 & 4 & B2 \\
8 & 5 & 5 & 3 & 2 & 4 & 4 & B2 \\
9 & 4 & 4 & 3 & 4 & 4 & 4 & B2 \\
10 & 5 & 5 & 4 & 5 & 5 & 5 & C1 \\
11 & 4 & 4 & 3 & 4 & 3 & 3 & B1 \\
12 & 5 & 4 & 2 & 2 & 2 & 3 & B1 \\
13 & 3 & 3 & 4 & 4 & 4 & 3 & B1 \\
14 & 6 & 6 & 5 & 6 & 6 & 6 & C2 \\
15 & 6 & 6 & 4 & 5 & 5 & 5 & C1 \\
16 & 5 & 5 & 5 & 4 & 5 & 5 & C1 \\
17 & 5 & 5 & 4 & 4 & 3 & 4 & B2 \\
18 & 5 & 6 & 4 & 5 & 4 & 5 & C1 \\
19 & 3 & 3 & 3 & 3 & 2 & 3 & B1 \\
20 & 6 & 6 & 4 & 3 & 5 & 5 & C1 \\
21 & 4 & 4 & 3 & 3 & 4 & 4 & B2 \\
22 & 3 & 5 & 3 & 4 & 4 & 4 & B2 \\
23 & 5 & 5 & 3 & 2 & 4 & 4 & B2 \\
24 & 4 & 4 & 3 & 4 & 4 & 4 & B2 \\
25 & 5 & 5 & 4 & 5 & 5 & 5 & C1 \\
\hline Total & 4 & 5 & 4 & 4 & 4 & 4 & B2 \\
\hline & & & & & & & \\
\hline
\end{tabular}

Del total de los datos obtenidos de la muestra de estudio respecto al nivel competencial digital docente, se concluye que, no se observan niveles bajos entre A1 o A2, considerados como niveles básicos. Por el contrario, los resultados muestran que el nivel más bajo fue el nivel B1 (Nivel intermedio bajo) y el más alto C2 (Nivel avanzado). En 
general, se puede observar que el promedio de los maestros de pedagogía de la actividad física y deporte respecto al nivel competencial digital docente es de B2 nivel intermedio alto. Esto, de acuerdo con lo establecido en el marco común de competencias digitales docentes en el INTEF (2017).

Los resultados alcanzados permitieron categorizar en frecuencias y porcentajes a la muestra total (tabla 4).

\section{Tabla 4}

Distribución por niveles en la muestra de estudio de docentes

\begin{tabular}{ccc}
\hline $\begin{array}{c}\text { Nivel de competencias } \\
\text { digitales }\end{array}$ & $\mathrm{f}$ & $\%$ \\
\hline B1 & 5 & 20.0 \\
B2 & 10 & 40.0 \\
C1 & 9 & 36.0 \\
C2 & 1 & 4.0 \\
\hline Total & 25 & 100.0 \\
\hline
\end{tabular}

Analizando los resultados se observó que el mayor porcentaje de docentes se encuentran en el nivel B2 que se caracteriza por poseer un nivel de competencia intermedio, por lo que, de forma independiente, responsable a sus necesidades y resolviendo problemas bien definidos, puede desarrollar su competencia digital.

Consiguientemente se valoró las dimensiones de estrategias digitales enunciadas por los grupos de estudiantes sobre el proceso docente (tabla 5).

\section{Tabla 5}

Valoración de las dimensiones de estrategias digitales en la muestra de estudiantes

\begin{tabular}{cccccccc}
\hline CI & $\begin{array}{c}\text { Estrategias } \\
\text { IAI }\end{array}$ & $\begin{array}{c}\text { Estrategias } \\
\text { CC }\end{array}$ & $\begin{array}{c}\text { Estrategias } \\
\text { CCD }\end{array}$ & $\begin{array}{c}\text { Estrategias } \\
\text { S }\end{array}$ & $\begin{array}{c}\text { Estrategias } \\
\text { RP }\end{array}$ & $\begin{array}{c}\text { Índice } \\
\text { de EA }\end{array}$ & $\begin{array}{c}\text { Nivel de } \\
\text { aplicación de EA }\end{array}$ \\
\hline GE1 & 17.0 & 12.8 & 37.3 & 8.4 & 12.7 & 88.1 & Medio \\
GE2 & 17.7 & 13.0 & 38.9 & 8.8 & 13.3 & 91.5 & Alto \\
GE3 & 17.8 & 13.2 & 37.8 & 8.4 & 13.0 & 90.2 & Medio \\
GE4 & 16.5 & 12.2 & 35.1 & 8.2 & 12.4 & 84.4 & Bajo \\
GE5 & 17.9 & 12.6 & 37.0 & 8.1 & 12.9 & 88.4 & Medio \\
GE6 & 17.4 & 12.6 & 35.6 & 8.0 & 12.4 & 85.9 & Bajo \\
GE7 & 18.2 & 13.2 & 39.2 & 8.6 & 13.1 & 92.2 & Alto \\
GE8 & 17.9 & 13.4 & 39.7 & 8.9 & 13.5 & 93.4 & Alto \\
GE9 & 17.0 & 12.7 & 36.3 & 8.3 & 12.7 & 87.0 & Bajo \\
\hline
\end{tabular}




\section{Tabla 5}

Valoración de las dimensiones de estrategias digitales en la muestra de estudiantes

\begin{tabular}{cccccccc}
\multicolumn{7}{c}{ (continuación) } \\
\hline CI & $\begin{array}{c}\text { Estrategias } \\
\text { IAI }\end{array}$ & $\begin{array}{c}\text { Estrategias } \\
\text { CC }\end{array}$ & $\begin{array}{c}\text { Estrategias } \\
\text { CCD }\end{array}$ & $\begin{array}{c}\text { Estrategias } \\
S\end{array}$ & $\begin{array}{c}\text { Estrategias } \\
\text { RP }\end{array}$ & $\begin{array}{c}\text { Índice } \\
\text { de EA }\end{array}$ & $\begin{array}{c}\text { Nivel de } \\
\text { aplicación de EA }\end{array}$ \\
\hline GE10 & 17.6 & 12.8 & 38.3 & 8.6 & 12.9 & 90.3 & Medio \\
GE11 & 18.1 & 13.5 & 39.0 & 8.7 & 13.6 & 92.8 & Alto \\
GE12 & 16.6 & 12.4 & 36.1 & 8.2 & 12.4 & 85.7 & Bajo \\
GE13 & 17.1 & 12.1 & 35.5 & 8.0 & 12.6 & 85.2 & Bajo \\
GE14 & 17.9 & 12.8 & 35.9 & 8.1 & 12.6 & 87.2 & Bajo \\
GE15 & 17.5 & 12.8 & 37.4 & 8.3 & 12.7 & 88.7 & Medio \\
GE16 & 18.3 & 13.6 & 40.8 & 9.1 & 13.7 & 95.5 & Alto \\
GE17 & 17.6 & 13.0 & 37.2 & 8.4 & 13.0 & 89.3 & Medio \\
GE18 & 16.9 & 12.5 & 37.3 & 8.4 & 12.5 & 87.6 & Medio \\
GE19 & 17.9 & 13.2 & 38.7 & 8.8 & 13.5 & 91.9 & Alto \\
GE20 & 17.5 & 12.9 & 37.7 & 8.6 & 13.1 & 89.7 & Medio \\
GE21 & 16.3 & 11.7 & 33.6 & 7.7 & 11.9 & 81.1 & Bajo \\
GE22 & 18.3 & 13.1 & 38.0 & 8.4 & 13.2 & 90.9 & Alto \\
GE23 & 17.4 & 12.6 & 35.8 & 7.9 & 12.2 & 85.8 & Bajo \\
GE24 & 18.1 & 13.5 & 40.3 & 9.0 & 13.5 & 94.4 & Alto \\
GE25 & 17.8 & 13.1 & 38.2 & 8.6 & 13.3 & 91.0 & Alto \\
\hline Total & 17.5 & 12.8 & 37.5 & 8.4 & 12.9 & 89.1 & Medio \\
\hline & & & & & & \\
\end{tabular}

Los resultados alcanzados y en base a los baremos establecidos específicamente para la muestra de estudio: 90.8 - 94.4 nivel alto; 87.5 - 90.7 nivel medio y 81.1 - 87.4 nivel bajo, los cuales se construyeron en base a la media grupal y su dispersión de la desviación estándar positiva y negativa, permitieron categorizan a la muestra de docentes en diferentes niveles de aplicación de estrategias de aprendizaje (tabla 6).

\section{Tabla 6}

Distribución por niveles de estrategias de aprendizaje declarado por los grupos de estudiantes

\begin{tabular}{ccc}
\hline Niveles & Frecuencia & Porcentaje \\
\hline Alto & 9 & 36.0 \\
Medio & 8 & 32.0 \\
Bajo & 8 & 32.0 \\
\hline Total & 25 & 100.0
\end{tabular}

Los niveles de aplicación de estrategias de aprendizaje con mayor prevalencia es el nivel alto, pues del total de 25 personas evaluadas, 9 representan el $36.0 \%$ en el nivel alto. 
Asimismo, la muestra de estudio refleja que, en el nivel medio y bajo se encuentran 8 personas representando el $32.0 \%$.

Valoradas ambas variables de estudio se procedió a realizar un análisis de relación (tabla 7).

\section{Tabla 7}

Relación entre niveles de competencias digitales y estrategias de aprendizaje docente

\begin{tabular}{ccccc}
\hline \multirow{2}{*}{$\begin{array}{c}\text { Nivel competencias } \\
\text { digitales }\end{array}$} & \multicolumn{2}{c}{ Nivel de estrategias de aprendizaje } & \multirow{2}{*}{ Total } \\
\cline { 2 - 4 } Alto & Medio & Bajo & \\
\hline B1 & 2 & 0 & 3 & 5 \\
B2 & 5 & 1 & 4 & 10 \\
C1 & 2 & 7 & 0 & 9 \\
C2 & 0 & 0 & 1 & 1 \\
\hline Total & 9 & 8 & 8 & 25 \\
\hline
\end{tabular}

Nota. Niveles de Competencias Digitales Docentes (CDD) y Niveles de Aplicación de Estrategias de Aprendizaje (EA).

En la tabla se observa que, cinco docentes están en el nivel B1 dos en nivel Alto y tres niveles bajo respecto a su competencial digital docente. De la misma manera, diez profesionales muestran estar en un nivel B2 cinco en nivel alto, nivel medio uno y bajo cuatro respecto a su competencia digital y los estudiantes afirmaron que dichos docentes respecto al nivel de aplicación de estrategias de aprendizaje oscilan entre un nivel bajo, medio y alto. Igualmente, respecto a los docentes nueve con nivel $\mathrm{C} 1$ avanzado, dos en nivel alto y siete en nivel medio. Finalmente, los educandos manifiestan que un docente con el nivel bajo en la competencial $\mathrm{C} 2$.

Respaldando el análisis descriptivo se realizó un proceso estadístico (tabla 8).

Tabla 8

Análisis estadístico de relación entre variables de estudio

\begin{tabular}{lccl}
\hline Estadísticos & Valor & $d f$ & $\begin{array}{l}\text { Significación } \\
\text { asintótica } \\
\text { (bilateral) }\end{array}$ \\
\hline Chi-cuadrado de Pearson & 16.478 & 6 & 0.011 \\
\hline \multicolumn{1}{c}{$\mathrm{N}$ de casos válidos } & & & 25 \\
\hline
\end{tabular}

La comprobación estadística a través de la prueba Chi-cuadrado de Pearson se basa en el valor crítico para el caso estudiado, en relación con el $95 \%$ de confiabilidad, 0.05 de rango de error y 6 grados de libertad (12.59), valor inferior al determinado en la presente investigación de16.478 que permite determinar que existe una relación entre las variables estudiadas, con el respaldo de la significación asintótica bilateral en un nivel de $\mathrm{P}<0,05$. 


\section{Conclusiones}

- Se valoró el nivel competencial digital docente de los maestros del área de Educación Física y se llegó a concluir que, se encuentran en un nivel competencial digital de B2 intermedio alto, por tanto, son capaces de mejorar su propia competencia digital y guiar a los estudiantes a desarrollar la suya. Igualmente, el área competencial digital docente dominante es el área de Información y Alfabetización Informacional, mientras que, en las áreas donde se encontraron los niveles más bajos corresponden a las áreas de Seguridad y Creación de Contenido Digital.

- Se identificó el nivel de aplicación de estrategias de aprendizaje de los docentes y se concluyó que, varía en función de los diferentes grupos en el que se imparte la docencia, puesto que, los niveles encontrados varían desde un nivel alto hasta un nivel bajo, seguido de un nivel medio.

- Se analizó la relación entre el nivel competencial digital docente y el nivel de aplicación de estrategias de aprendizaje en los estudiantes universitarios de formación en Educación Física y se encontró dicha relación a nivel descriptivo y estadístico. Por lo cual, los maestros que demostraron tener un alto nivel de competencias digitales efectivamente muestran un alto nivel de aplicación de estrategias de aprendizaje y viceversa.

\section{Referencias Bibliográficas}

Addine, F., Recarey, S., Fuxá, M., \& Fernández, S. (2020). Didáctica: teoría y práctica. Editorial Pueblo y Educación.

Al-Quraishi, A. M. (2021). Teaching and learning in university teaching and its importance. Ishraqat tanmawia, 7(27). Butcher, N. (2019). Marco de competencias docentes en materia de TIC UNESCO.

Cabero-Almenara, J., \& Palacios-Rodríguez, A. (2020). Marco Europeo de Competencia Digital Docente «DigCompEdu». Traducción y adaptación del

Casanova, I. I. (2018). Visión general del enfoque por competencias en Latinoamérica. Cedeño-Escobar, M. R., Ponce-Aguilar, E. E., Lucas-Flores, Y. A., \& PereroAlonzo, V. E. (2020). Classroom y Google Meet, como herramientas para fortalecer el proceso de enseñanza-aprendizaje. Polo del Conocimiento, 5(7), 388405.

Cuartero, M. D., Espinosa, M. P., \& Porlán, I. (2019). Certificación de la Competencia Digital Docente: propuesta para el profesorado universitario. RIED. Revista Iberoamericana de Educación a Distancia, 22(1). 
Del Rocío Peralvo, C. A. (2018). Retos de la docencia universitaria en el siglo XXI. Revista Órbita Pedagógica. ISSN 2409-0131, 5(1), 09-27.

Dussel, I. (2020). La formación docente y los desafíos de la pandemia. Revista Científica EFI. DGES Volumen, 6(10).

Engen, B. K. (2019). Comprendiendo los aspectos culturales y sociales de las competencias digitales docentes. Comunicar: Revista Científica de Comunicación y Educación, 27(61), 9-19.

Fernández, J. T., \& Pérez, K. (2018). Nuevos escenarios y competencias digitales docentes: hacia la profesionalización docente con TIC. Profesorado, revista de currículum y formación del profesorado, 22(1), 25-51.

Hernández, J. A., \& Ortiz, A. M. (2019). Formación docente: aportaciones para un estado del arte desde una perspectiva participativa. Revista Investigación Cualitativa, 4(1), 75- 90 .

Instituto Nacional de Tecnologías Educativas y Formación del Profesorado [INTEF]. (2017). Competencia digital docente octubre 2017.

Loaiza-Aguirre, M. I., \& Andrade-Abarca, P. S. (2021). Análisis de programas de desarrollo profesional del profesorado universitario. Revista Virtual Universidad Católica del Norte., 63, 161-195.

Macanchí Pico, M. L. (2020). Innovación educativa, pedagógica y didáctica. Concepciones para la práctica en la educación superior. Revista Universidad y Sociedad., 12(1), 396-403.

Martínez, M. C. (2020). Caracterización del perfil de las competencias laborales en el docente universitario. Orbis: revista de Ciencias Humanas, 15(45), 23-37.

Otero, L. C., Cerqueiras, E., Fernández, R., \& Antelo, B. (2021). Competencia Digital Docente del profesorado de FP de Galicia. Píxel-Bit. Revista de Medios y Educación., 61, 165-196.

Parra González, M. E., \& Segura Robles, A. (2019). Producción científica sobre gamificación en educación: Un análisis cienciométrico. Revista de educación.

Polo, F. A. (2020). Pedagogía afectiva para dinamizar el aprendizaje significativo en tiempos de emergencia sanitaria. Revista Ibérica de Sistemas e Tecnologías de Informação, (E40), 166-179. 
Ramírez-García, A. (2018). Las competencias docentes genéricas en los grados de educación. Visión del profesorado universitario. Estudios pedagógicos (Valdivia)., 44(2), 259-277.

Sandoval, A. R., \& Guajardo, M. (2017). Análisis documental del perfil competencial del docente universitario de un país latinoamericano de ingresos medios, mediante teoría fundamentada. CIAIQ 2017, 1.

Santos-Loor, C. E., Vélez-Loor, J. M., Aguilera-Meza, C. K., \& Bowen-Rivera, A. C. (2021). La Educación Ecuatoriana vs la Pandemia del Covid-19. Dominio de las Ciencias, 7(2), 105-124.

Suárez, N. (2020). Formación docente universitaria y crisis sanitaria COVID-19. CienciAmérica, 9(2), 109-114. 
El artículo que se publica es de exclusiva responsabilidad de los autores y no necesariamente reflejan el pensamiento de la Revista Conciencia Digital.

\section{Ciencia}

El artículo queda en propiedad de la revista y, por tanto, su publicación parcial y/o total en otro medio tiene que ser autorizado por el director de la Revista Conciencia Digital.
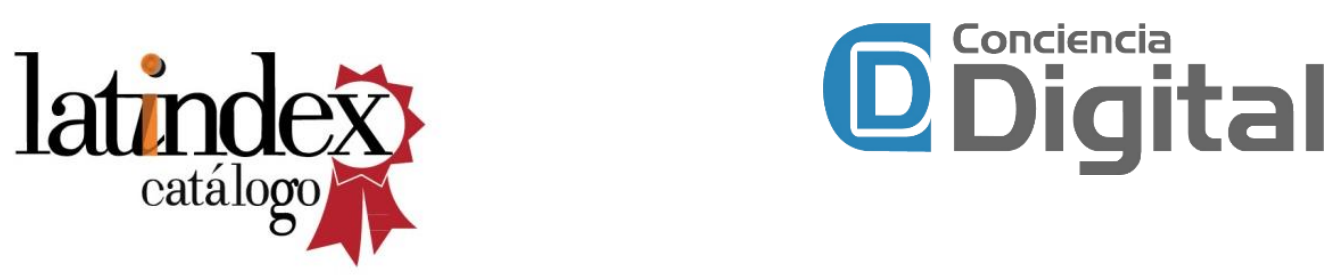

Indexaciones

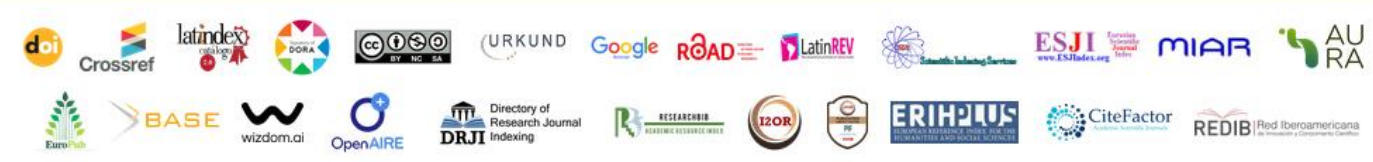

